\title{
Seroprevalence and Molecular Epidemiosurveillance of Brucellosis in Pakistan
}

\author{
Usman Waheed ${ }^{\star 1}$, Zargham Nawaz Butt ${ }^{2}$, Waqas O. Ashraf ${ }^{3}$ and Qaiser Mahmood Khan ${ }^{2}$
}

${ }^{1}$ Microbiology, Pathobiology, University of Veterinary \& Animal Sciences, Lahore, Pakistan, Lahore, Pakistan; ${ }^{2}$ National Institute for Biotechnology \& Genetic Engineering, Faisalabad, FAISALABAD, Pakistan; ${ }^{3}$ Islamia University, Bahawalpur, Bahawalpur, Pakistan

\section{Objective}

To detect the presence of brucella in serum samples of occupationally exposed human and animals by conventional screening methods.

To perform epidemiosurvelliance of brucella molecular based tests including genus and species specific PCR.

To check the brucella prevalence in occupationally exposed human.

\section{Introduction}

Livestock sector contributes more than $58 \%$ to agriculture-based economy of Pakistan. Diseases of socio-economic importance are posing an enormous pressure to the growth of this sector. Zoonotic diseases are generally neglected in wake of epizootics having epidemic potential. One Health is a multi-sectoral approach to control zoonotic diseases at animal level to mitigate risk of transfer to the humans and environment. Despite various control programs, zoonosis is known to cause public health emergencies at various regional and national levels. OIE declared brucellosis as a model bacterial disease to control zoonosis in developing countries. Genus Brucella is expanding with its discovery in various amphibian species and marine mammals and demands control efforts at various levels. Reporting of zoonosis is less than actual prevalence in third world countries like Pakistan where disease is considered endemic but no official data is available. In this study, brucellosis was used as a model disease to emphasize the significance of One Health.

\section{Methods}

In total, 183 occupationally exposed human and 324 animal blood samples were collected from five different geographical areas of Punjab and one region from KP. For detection of brucells, rose bangal plate test (RBPT) and cELISA were carried out on serum samples. For molecular epidemiosurveillance genus specific PCR BCSP31 and specie specific PCR IS711 were conducted. Fifty-seven milk samples as environmental samples were aslo collected. For the testing of milk for the detection of brucella, Milk Ring Test (MRT) was applied.

\section{Results}

Serologically in animals $26(8 \%)$ samples were found positive by RBPT \& 31(9\%) by cELISA. Disease was detected in $42(13 \%)$ \& $59(18 \%)$ samples by applying molecular methods using genus specific PCR BCSP 31 \& specie specific PCR IS711. Disease was recorded in humans as $16(8 \%), 24(13 \%), 33(18 \%), 56(30 \%)$ by RBPT, cELISA, PCR BCSP31 \& PCR IS711, respectively. Out of 57 milk samples collected from different areas were tested by Milk Ring Test (MRT) $\& 12(21 \%)$ samples were found positive.

\section{Conclusions}

It is a significant finding that raw milk is a constant source of disease exposure to farmers, milking men and general users. Disease prevalence was more in people associated with milking activities possibly due to use of raw milk. This study validate the prevalence of brucellosis in Pakistan with significant presence of disease in occupationally exposed individuals emphasizing the close collaboration between veterinary and human health sectors.
This study will broaden our knowledge of disease prevalence and epidemiology in Pakistan. The data produced from this study will help in future control and eradication of this important zoonosis using one health approach.

\section{Keywords}

Brucellosis; Occupationally exposed; Pakistan; animals

\section{*Usman Waheed}

E-mail: dr.usman.waheed@gmail.com 